\title{
Human Rhinovirus Infection Enhances the Th2 Environment in Allergic and Non-allergic Patients With Chronic Rhinosinusitis
}

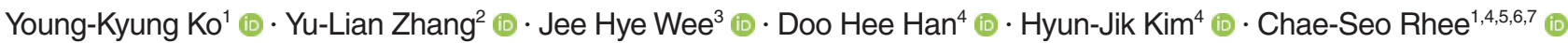 \\ ${ }^{1}$ Graduate School of Medicine, Seoul National University College of Medicine, Seoul, Korea, ${ }^{2}$ Center of Morphological Experiment, Medical \\ College of Yanbian University, Yanji, China; ${ }^{3}$ Department of Otorhinolaryngology-Head and Neck Surgery, Hallym University Sacred Heart \\ Hospital, Hallym University College of Medicine, Anyang, ${ }^{4}$ Department of Otorhinolaryngology-Head and Neck Surgery, Seoul National \\ University Hospital, Seoul National University College of Medicine, Seoul; ${ }^{5}$ Department of Otorhinolaryngology-Head and Neck Surgery, \\ Seoul National University Bundang Hospital, Seoul National University College of Medicine, Seongnam; ${ }^{6}$ Sensory Organs Research Center, \\ Seoul National University Medical Research Center, Seoul; ${ }^{7}$ Institute of Allergy and Clinical Immunology, Seoul National University Medical
} Research Center, Seoul, Korea

Objectives. This study was conducted to determine whether patients with allergic rhinitis might be more susceptible to human rhinovirus (HRV) infection and whether the effects of infection on the elicited immune responses are different in allergic and non-allergic patients with chronic rhinosinusitis (CRS).

Methods. Uncinate process tissues were obtained from 61 CRS patients (of whom 39 had allergies and 22 did not) and were infected with HRV-16 using an air-liquid interface organ culture system. The expression levels of programmed cell death-ligand (PD-L)1, PD-L2, intracellular adhesion molecule 1, interferon-gamma (IFN- $\gamma$ ), interleukin (IL)-4, IL-5, and IL-10 were evaluated in the infected nasal mucosa.

Results. The HRV infection rates were not significantly different between the allergy (74.4\%) and non-allergy $(72.7 \%)$ groups. In the allergy group, the expression of PD-L1 $(P=0.013)$ and IL-10 $(P=0.040)$ was significantly elevated in the HRV-infected tissues, and there was a strong correlation between PD-L1 and IL-10 $(r=0.868, P<0.001)$. In contrast, infected tissues from the non-allergy group displayed increased levels of IL-4 $(P=0.039)$, IL-5 $(P=0.023)$, and IFN- $\gamma(P=0.031)$, as well as an increased IL-4/IFN- $\gamma$ ratio, after HRV infection $(P=0.043)$.

Conclusion. This study showed that HRV infection rates were similar in the nasal mucosa of patients with CRS regardless of the presence of allergic rhinitis. HRV infection enhanced the Th2 environment by modulating PD-L1 and PD-L2 expression levels in allergic mucosa and by increasing the IL-4/IFN- $\gamma$ ratio in non-allergic mucosa.

Keywords. Rhinovirus; Respiratory Tract Infection; Allergic Rhinitis; Sinusitis; B7-H1 Antigen

\section{INTRODUCTION}

- Received March 25, 2020

Revised August 31, 2020

Accepted September 9, 2020

- Corresponding author: Chae-Seo Rhee

Department of Otorhinolaryngology-Head and Neck Surgery, Seoul

National University Hospital, 101 Daehak-ro, Jongno-gu, Seoul 03080,

Korea

Tel: +82-2-2072-3991, Fax: +82-2-766-2447

E-mail: csrhee@snu.ac.kr
Human rhinovirus (HRV) is a common viral pathogen associated with upper respiratory infections; it initially infects the nasal and nasopharyngeal epithelial cells. HRV is a small, non-enveloped, positive-sense, and single-stranded RNA virus belonging to the Picornaviridae family [1-3]. Monocytes, macrophages, and fibroblasts can be infected by HRV, and the airway epithelial cells are the major sites of HRV infection. On their surface, epithelial

Copyright () 2021 by Korean Society of Otorhinolaryngology-Head and Neck Surgery.

This is an open-access article distributed under the terms of the Creative Commons Attribution Non-Commercial License (https://creativecommons.org/licenses/by-nc/4.0)

which permits unrestricted non-commercial use, distribution, and reproduction in any medium, provided the original work is properly cited. 
cells express intracellular adhesion molecule 1 (ICAM-1) or lowdensity lipoprotein receptors, which are the sites of HRV attachment. HRV induces the expression of a wide range of pleiotropic cytokines, growth factors, and chemokines [1,4].

Furthermore, there are no signs of declining trends in the prevalence of atopic diseases in many parts of the world. It is wellestablished that bacterial and viral infections exacerbate allergic rhinitis (AR) and other atopic diseases. The interaction between viral infections and allergies is complex, and the mechanism remains unclear. However, some studies have suggested that coregulatory molecules and cytokines might play a role $[5,6]$.

Viral modulation of an inhibitory molecule of the B7 family in epithelial cells results in suppression or termination of the immune response. Programmed cell death-ligand 1 (PD-L1, B7-H1, CD274) and (PD-L2, B7-DC, CD273) belong to the B7 family and are widely expressed on cells such as activated T cells, B cells, monocytes, dendritic cells, and macrophages to modulate activation or inhibition. They are also found in non-lymphoid tissues including the airway epithelial cells, suggesting that they may modulate local tissue T cell functions. Binding of PD-L1 and PD-L2 to their receptor, programmed cell death 1 (PD-1), negatively regulates $\mathrm{T}$ cells by decreasing their proliferation and the production of effector cytokines. In addition, PD-1 activity is required for the termination of the late phase of allergic inflammation [6-9].

In macrophages, interleukin (IL)-4 induces PD-L2 more preferentially than interferon-gamma (IFN- $\gamma$ ), whereas IFN- $\gamma$ induces PD-L1 more preferentially than IL-4, suggesting the differential mobilization of PD-L1 and PD-L2 by the Th1 and Th2 responses. Acting via a negative feedback loop, upregulation of PD-L2 by IL-4 suppresses IL-4 production and modulates the severity of asthma. Loss of PD-L2 in this PD-L2/IL-4 circuit results in increased IL-4 and airway hyperresponsiveness (AHR). Similarly, enhanced PD-L1 activity inhibits IFN- $\gamma$ via a negative feedback loop and increases AHR. As a result, loss of PD-L1 in this PD-L1/IFN- $\gamma$ circuit results in increased IFN- $\gamma$ and diminished AHR [9-11].

PD-L1 binds to PD- 1 and triggers IL-10 production $[9,12]$. IL10 is known to be a major anti-inflammatory cytokine with the important function of controlling diverse immune responses, such as preventing autoimmune and allergic inflammatory re-

\section{H I G H L I G G H T S}

- Human rhinovirus (HRV) infection rates in the allergy (74.4\%) and non-allergy ( $72.7 \%$ ) groups were comparable.

- In the allergy group, HRV infection modulated programmed cell death-ligand (PD-L) 1 and PD-L2 to enhance the Th2 environment.

- In non-allergy group, HRV infection increased the ratio of interleukin-4 to interferon-gamma to induce Th2 cytokines. sponses $[13,14]$. It has the ability to suppress the function of antigen-presenting cells, to downregulate the function of dendritic cells, and to prevent the development of allergen-induced airway inflammation and AHR. However, IL-10 also stimulates Th2 cells and mast cells and promotes B cell maturation and antibody production [15].

Previous studies have suggested diverse opinions about the relationship between HRV infection and allergic reactions. Viral infection has both positive and negative effects on the development of airway allergies via the inhibition and enhancement of allergic sensitization $[6,10]$. This study was conducted to determine the susceptibility of HRV infection and the effects of HRV infection on the immune response in allergic and non-allergic patients with chronic rhinosinusitis (CRS).

\section{MATERIALS AND METHODS}

\section{Study subjects}

The uncinate precess (UP) mucosal tissues were obtained from 61 patients who underwent surgical management of CRS at the Seoul National University Hospital and Seoul National University Bundang Hospital, Korea. Of these, 39 patients had AR and 22 did not. Individuals, who had viral upper respiratory infection within 6 weeks and had been treated with medication, were excluded from the study. The design of this study was approved by the Institutional Review Board of Seoul National University Hospital (IRB No. 1403-026-563) and Seoul National University Bundang Hospital (IRB No. B-1209/171-001), and all enrolled subjects provided written informed consent.

The demographics of the $39 \mathrm{AR}$ and 22 non-AR subjects are outlined in Table 1 . The presence of allergy was confirmed by skin prick test (Allergopharma, Reinbek, Germany) and the presence of allergic symptoms.

Table 1. Demographics of AR and non-AR subjects

\begin{tabular}{lccc}
\hline Characteristics & AR $(n=39)$ & Non-AR $(n=22)$ & $P$-value \\
\hline Sex & & & 0.415 \\
$\quad$ Male & 26 & 12 & \\
$\quad$ Female & 13 & 10 & \\
Diagnosis & & & 0.587 \\
$\quad$ CRSsNP & 25 & 12 & \\
CRSwNP & 14 & 10 & \\
Age $(\mathrm{yr})$ & $43.54 \pm 17.45$ & $36.14 \pm 20.68$ & 0.142 \\
Serum eosinophils count & $314.8 \pm 293.9$ & $153.7 \pm 94.81$ & $0.006^{*}$ \\
$\quad$ cells/ $\mu \mathrm{L})$ & & & \\
Serum ECP $(\mu \mathrm{g} / \mathrm{L})$ & $25.57 \pm 31.70$ & $15.69 \pm 13.39$ & 0.141 \\
Serum total $\mathrm{IgE}(\mathrm{U} / \mathrm{mL})$ & $277.4 \pm 440.41$ & $42.22 \pm 67.76$ & $<0.001^{* *}$ \\
\hline
\end{tabular}

Values are presented as mean \pm standard deviation.

AR, allergic rhinitis; CRSsNP, chronic rhinosinusitis without nasal polyps; CRSwNP, chronic rhinosinusitis with nasal polyps; ECP, eosinophil cationic proteins; IgE, immunoglobulin $\mathrm{E}$.

${ }^{*} P<0.01 .{ }^{\star} P<0.001$. 


\section{HRV-16 infection in organ culture}

HRV-16 was purchased from the American Type Culture Collection (ATCC) and was grown in HeLa cells (ATCC) to determine the $50 \%$ tissue culture infectious dose $\left(\mathrm{TCID}_{50}\right)$ for virus titration. In addition, by ultraviolet (UV) inactivation of HRV, we confirmed that all HRV mediated responses were specific to the active virus (data not shown).

The UP tissues were washed with normal saline solution to remove the blood cells and mucus, and then incubated overnight at $4^{\circ} \mathrm{C}$ in minimal essential medium supplemented with $2 \mathrm{mM}$ L-glutamine, $20 \mathrm{mM}$ hydroxyethyl piperazine ethane sulfonicacid (HEPES), penicillin $(100 \mathrm{U} / \mathrm{mL})$, streptomycin $(100 \mu \mathrm{g} / \mathrm{mL})$, and amphotericin B $(0.2 \mu \mathrm{g} / \mathrm{mL}$; Thermo Fisher, Grand Island, NY, USA). Tissues were cut into several pieces and placed on a multicel (Kwang Woo Medix Inc., Seoul, Korea) soaked with culture medium. The multicel, a polyvinyl acetal surgical sponge, had no effect on the nasal tissue. In the air-liquid interface organ culture system [16,17], the nasal tissues are in contact with the air on the multicel, whereas the culture medium supplies nutrients to the submucosal area of the nasal mucosa through the multicel soaked in media. One uncinate process mucosa was cut into several pieces that were then divided into three groups and treated with medium (control), replication-deficient avirulent HRV16 that was exposed to UV, or HRV16 $\left(10^{5} \mathrm{TCID}_{50} \mathrm{U} / \mathrm{mL}\right)$. The epithelium of each tissues was supplied with medium, avirulent HRV16, or HRV16 for 4 hours at $33^{\circ} \mathrm{C}$ then, the pieces were washed six times with PBS to remove the previously applied HRVs. To ascertain that no HRVs remained on the surface of tissues, we assayed the HRVs in the sixth PBS washing solution using semi-nested reverse transcription polymerase chain reaction (RT-PCR). Next, the mucosae were transferred to a new plate and incubated for 48 hours at $33^{\circ} \mathrm{C}$.

\section{Determination of successful HRV infection}

When nasal mucosa tissues are infected with HRV, viral replication takes places inside the cells, and the HRVs are released to the cell surface. To determine whether the tissues were infected, we first washed the specimen with PBS to obtain the surface fluid; then, total RNA was extracted from the surface fluid using Trizol LS reagent (Thermo Fisher) according to the manufacturer's protocol. In RT-PCR, the extracted RNA was reverse transcribed and amplified using the primers OL26 (5'-GCACTTCTGTTTCCCC-3') and OL27 (5'-CGGACACCCAAAGTAG-3') for semi-nested PCR; the RT-PCR product was amplified using the primers OL26 and JWA-1b (5'-CATTCAGGGGCCGGAGGA-3'). Successful infection was confirmed by identifying a 292bp HRV specific band [16].

\section{PD-L1, PD-L2, and cytokine mRNA in the nasal mucosa}

Total RNA was extracted and purified from the nasal mucosa tissue using Trizol LS reagent (Thermo Fisher) according to the manufacturer's protocol. The extracted RNA was reverse tran- scribed into cDNA using amfiRivert Platinum cDNA synthesis master mix (GenDEPOT, Katy, TX, USA) with a thermal cycler. Real- time PCR was used to detect PD-L1, PD-L2, ICAM-1, IFN- $\gamma$, IL-4, IL-5, and IL-10 gene expression. The comparative cycle of threshold method was used to demonstrate the relative levels of the target genes.

\section{PD-L1, PD-L2, ICAM-1, and IL-10 protein expression in the nasal mucosa}

PD-L1, PD-L2, ICAM-1, and IL-10 proteins were measured by Western blot analysis using anti-PD-L1 (Abcam, Cambridge, MA, USA), anti-PD-L2 (R\&D systems, Minneapolis, MN, USA), anti-ICAM-1 (Cell Signaling Technology, Danvers, MA, USA), anti-IL-10 (Abcam), and anti- $\beta$-actin (Cell Signaling Technology) antibodies, respectively. Western blots were visualized using Super Signal West Pico Chemiluminescent Substrate (Thermo Fisher). The quantitative analysis of signal intensity was performed using Alpha View SA software (Protein simple, San Jose, CA, USA).

\section{Statistical analysis}

Data are presented as the mean \pm standard error of the mean. Differences between the allergy and non-allergy groups were analyzed using the Mann-Whitney test, unpaired $t$-test or Fisher's exact test. The Wilcoxon matched pairs test or the paired $t$-test was used for comparisons between control and the HRVinfected tissues. The Spearman correlation coefficient test was used to evaluate the correlations between PD-L1 and IL-10 in the allergy and non-allergy groups. A $P$-value less than 0.05 was considered statistically significant. GraphPad Prism version 5.0 (GraphPad Software, San Diego, CA, USA) was used for the statistical analysis and graphing.

\section{RESULTS}

\section{$\mathrm{HRV}$ infection in the allergy and non-allergy groups}

Among the 22 uncinate process tissues from the non-allergy group, 16 tissues $(72.7 \%$ ) showed a HRV-specific band. Of the 39 uncinate process tissues from the allergy group, 29 mucosae (74.4\%) were successfully infected with HRV.There was no significant difference between the groups $(P=1.0$, Fisher's exact test).

\section{Different responses to HRV infection according to the presence or absence of $A R$ Expression of PD-L1 and PD-L2 in the nasal mucosa}

The level of PD-L1 mRNA was significantly elevated in the HRV-infected nasal tissue, both in the allergy group $(P=0.048)$ and the non-allergy group $(P=0.027)$; however, increased expression of the PD-L1 protein was evident only in the infected nasal tissue from the allergy group $(P=0.013)$ (Fig. $1 \mathrm{~A})$. In con- 

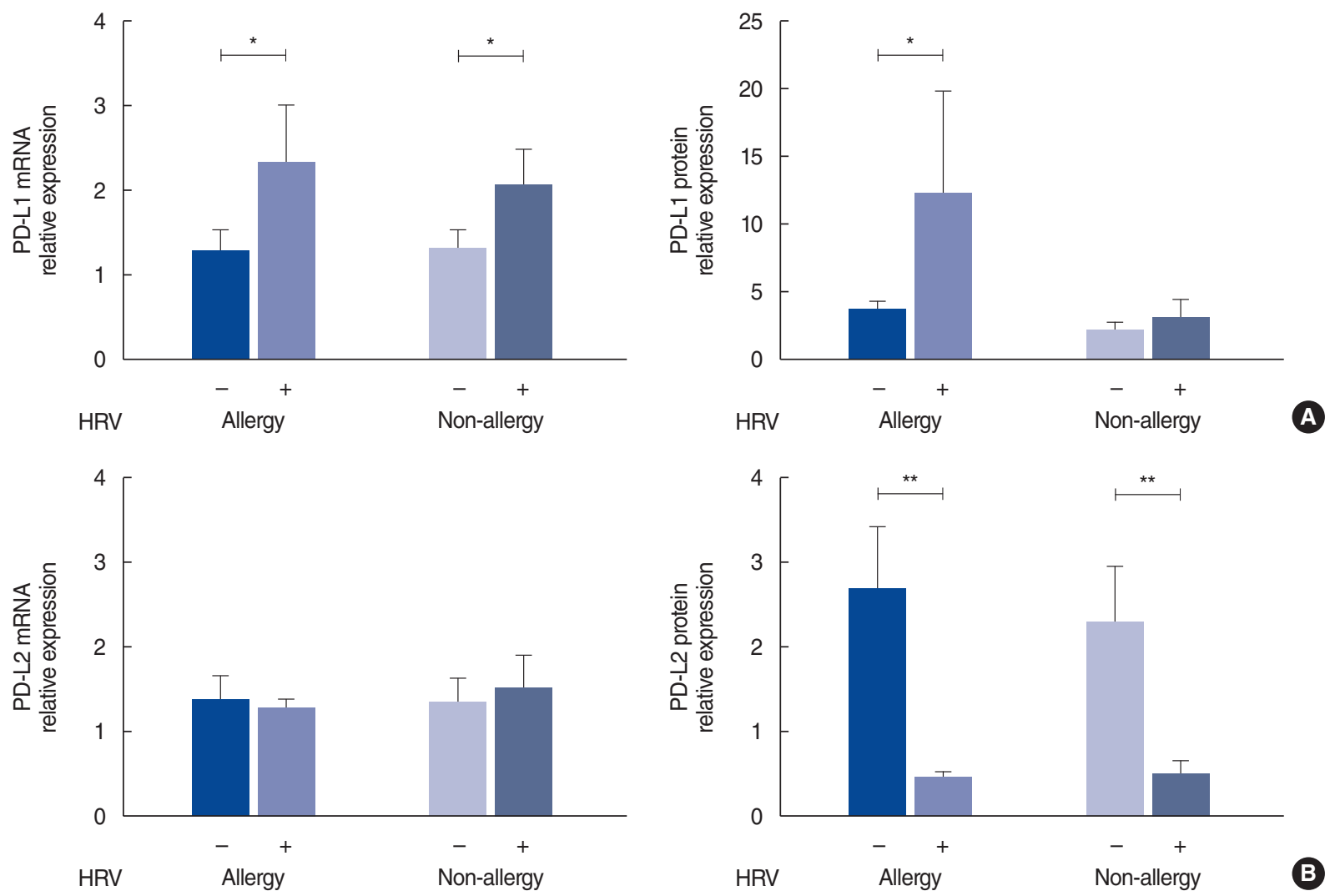

Fig. 1. Programmed cell death-ligand (PD-L)1 and PD-L2 expression after human rhinovirus (HRV) infection in the allergy and non-allergy groups. (A) mRNA and protein levels of PD-L1 after HRV infection (B) mRNA and protein levels of PD-L2 after HRV infection. The relative expression of mRNA was calculated based on the $\triangle \triangle C_{\text {T }}$ method; the relative expression of protein represents the ratio of PD-L1 or PD-L2 to $B$-actin. Values are presented as mean \pm standard error of the mean. ${ }^{\star} P<0.05$, ${ }^{\star \star} P<0.001$.
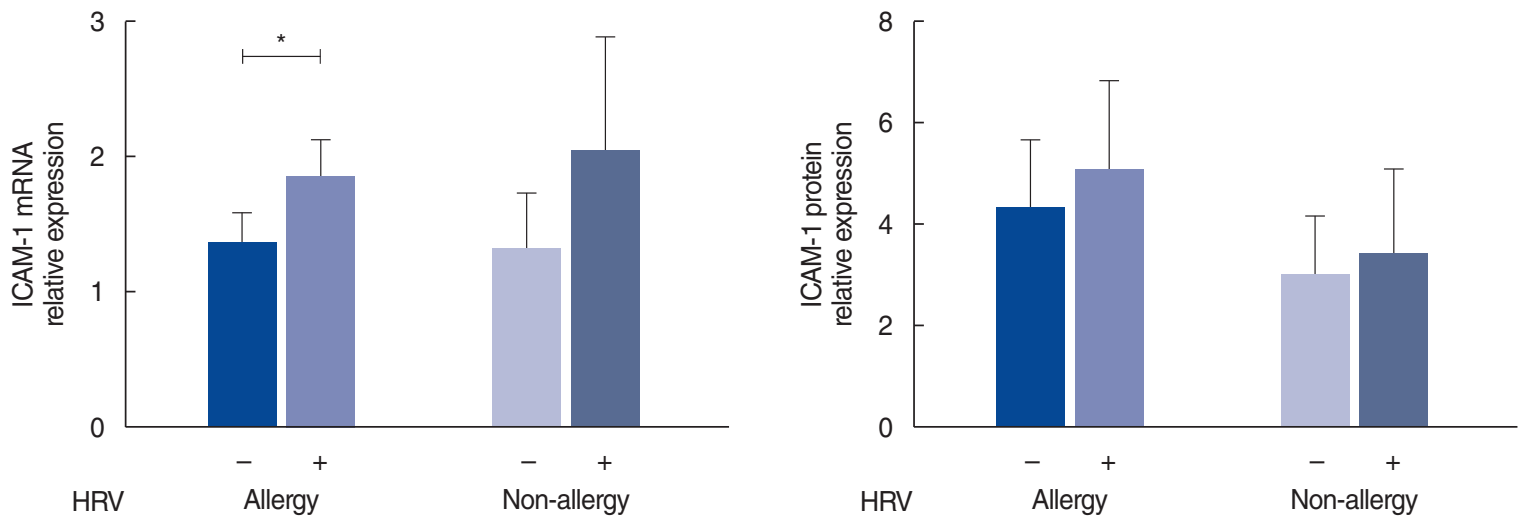

Fig. 2. Intracellular adhesion molecule 1 (ICAM-1) expression after human rhinovirus (HRV) infection in the allergy and non-allergy groups. mRNA and protein levels of ICAM-1 after HRV infection. The relative expression of mRNA was calculated based on the $\triangle \triangle C_{T}$ method; the relative expression of protein represents the ratio of ICAM- 1 to $B$-actin. Values are presented as mean \pm standard error of the mean. ${ }^{\star} P<0.05$.

trast, the PD-L2 mRNA levels were not significantly different in either group, in both the control and HRV-infected tissues (Fig. 1B). The HRV-infected tissue showed significantly lower amounts of PD-L2 protein expression than the control tissue, both in the allergy $(P=0.003)$ and non-allergy groups $(P=0.010)$.

\section{Expression of ICAM-1 in the nasal mucosa}

In the allergy group, ICAM-1 mRNA levels were slightly higher in the infected tissues $(P=0.022)$; however, ICAM-1 protein expression did not show a statistically significant difference (Fig. 2). In the non-allergy group, the mRNA and protein levels of ICAM-1 were not significantly different between the control 

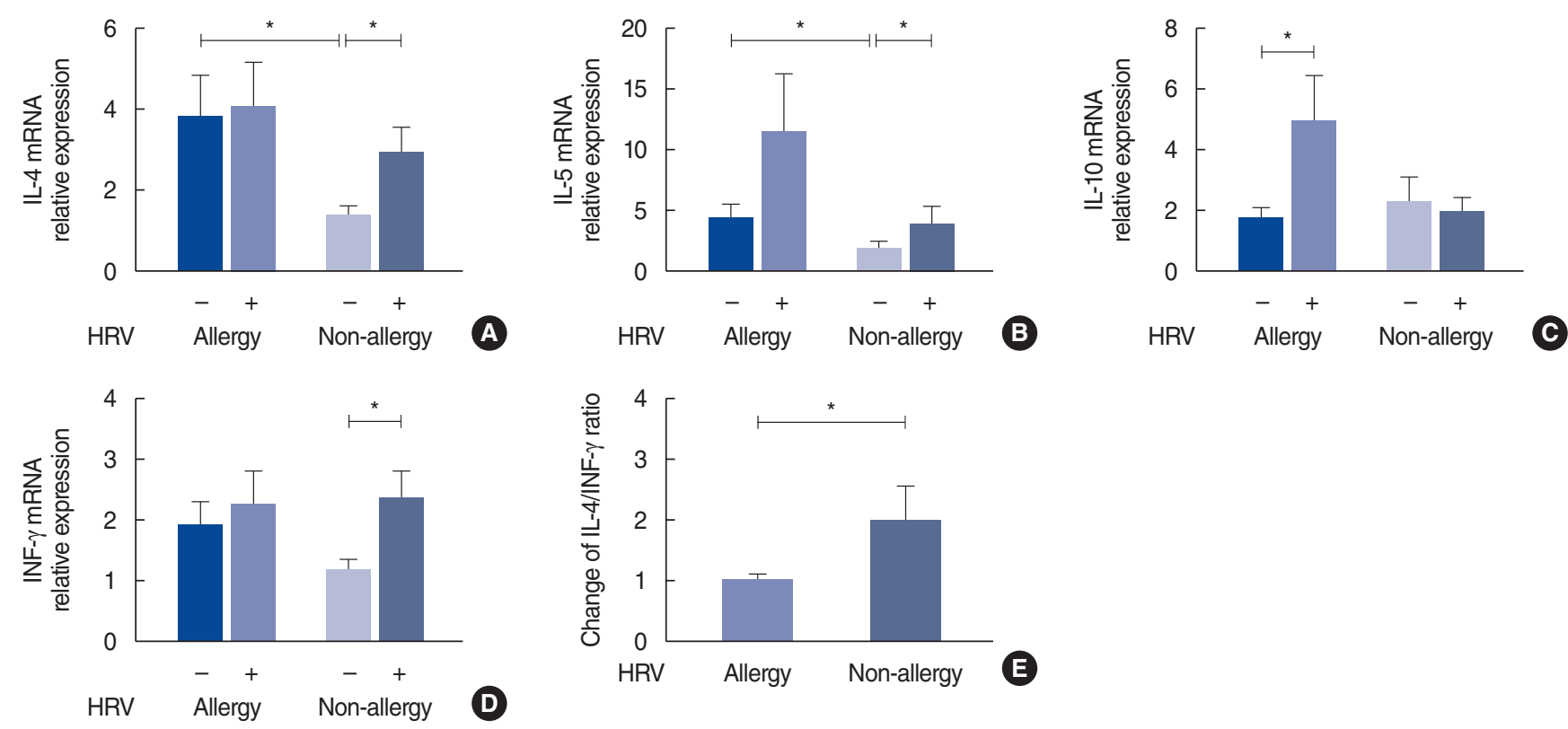

Fig. 3. Interleukin (IL)-4, IL-5, IL-10 and interferon (IFN) levels after human rhinovirus (HRV) infection in the allergy and non-allergy groups. (A) IL-4 mRNA, (B) IL-5 mRNA, (C) IL-10 mRNA, (D) IFN- $\gamma$ mRNA levels after HRV infection. (E) Change in the IL-4/INF- $\gamma$ ratio after HRV infection in each subject. The relative expression of mRNA was calculated based on the $\Delta \Delta \mathrm{C}_{\mathrm{T}}$ method. Values are presented as the mean \pm standard error of the mean. ${ }^{\star} P<0.05$.

and HRV-infected tissues. Additionally, when comparing the allergy and non-allergy groups, no significant difference was observed between their mRNA and protein expression levels.

Expression of IL-4, IL-5, IL-10, and IFN- $\gamma$ in the nasal mucosa HRV infection significantly increased the level of IL-10 $(P=0.040)$ in the allergy group, whereas in the non-allergy group, HRV infection increased the levels of IL-4 $(P=0.039)$, IL-5 $(P=0.023)$ and IFN- $\gamma(P=0.031)$ (Fig. 3). The infected mucosa in the allergy group tended to show an increase in the level of IL-5, but, the difference was not statistically significant. Similarly, in the allergy group, the levels of IL-4 and IFN- $\gamma$ did not increase significantly. When the allergy and non-allergy groups were compared, the control mucosa from the allergy group showed significantly higher IL-4 $(P=0.035)$ and IL-5 $(P=0.043)$ mRNA levels than those from the non-allergy group. In Fig. 3E, the Y-axis of the graph represents the IL-4/INF- $\gamma$ ratio in HRV-infected tissue over the IL-4/IFN- $\gamma$ ratio in control tissue in each subject. In the allergy group, the IL-4/IFN- $\gamma$ ratio did not change significantly after HRV infection. In contrast, in the non-allergy group, the IL-4/IFN- $\gamma$ ratio significantly increased after HRV infection $(P=0.043)$.

\section{Association between PD-L1 and IL-10 in the nasal mucosa}

We used the Spearman correlation coefficient test to evaluate correlations in the allergy and non-allergy groups. There was a strong correlation between PD-L1 and IL-10 in the allergy group $(r=0.868, P<0.001)$. In contrast, no correlation was observed in the non-allergy group $(r=-0.018, P=0.973)($ Fig. 4$)$. In the aller-

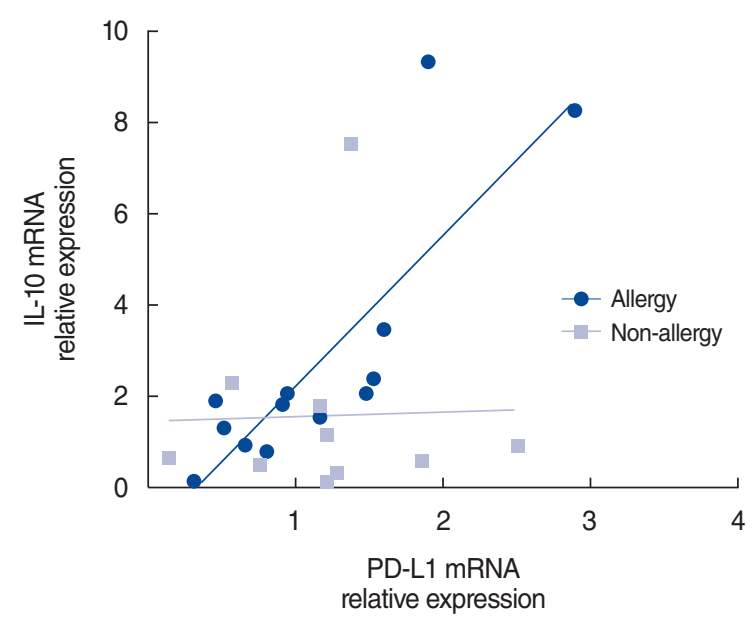

Fig. 4. Correlation of programmed cell death-ligand (PD-L)1 and interleukin (IL)-10 after human rhinovirus (HRV) infection. PD-L1 and $\mathrm{IL}-10$ mRNA levels after HRV infection in the allergy and non-allergy groups. The relative expression was calculated as the ratio of the expression in the infected to control tissue in each individual.

gy group, the increase in PD-L1 after HRV infection was likely associated with an increase in IL-10.

\section{DISCUSSION}

There is limited evidence that patients with AR have upper respiratory infections (common colds) more frequently than indi- 
viduals without $A R$. In this study, we aimed to determine whether patients with allergies might be more susceptible to HRV infection and whether the effects of infection on the immune response are different in allergic and non-allergic patients.

For a better understanding of the pathophysiology of HRV infection, an ideal experimental model is essential. In this study, we used an air-liquid interface organ culture system that appears to be similar to the real circumstances of HRV infection [16]. When HRVs infect the nasal epithelium, viral replication takes place; accordingly, HRVs are released to the cell surface, and can then be detected in the surface fluid of the nasal mucosa [5]. Some studies have shown that patients with atopic diseases are more vulnerable to infection, whereas other researchers have found no such correlations $[18,19]$. Patients with asthma have a higher frequency and severity of lower respiratory tract infections than those without asthma [19]. In addition, the atopic environment is associated with an increased risk of infection; however, the risks are affected by the type of virus, the host's immune genetics, and environmental factors $[5,20,21]$. In contrast, other studies reported that patients with asthma and those with atopic diseases did not appear to have a higher frequency and severity or longer duration of respiratory tract infections [22,23]. However, there is limited evidence that allergic CRS patients are more susceptible to common colds than non-allergic CRS patients. In our experimental results, tissues from $74.4 \%$ of allergic patients and $72.7 \%$ of non-allergic patients were successfully infected by HRV. Under chronic inflammatory conditions, allergic and nonallergic subjects had a similar HRV infection rate. This result suggests that an allergy itself may not increase vulnerability to HRV infection under chronic inflammatory conditions such as CRS.

Various conditions and many molecules affect rhinovirus infection, and HRV infection also regulates the expression of a wide range of factors. To determine the response to infection in the allergy and non-allergy groups, we investigated the levels of PD-L1, PD-L2, ICAM-1, IL-4, IL-5, IL-10, and IFN- $\gamma$ in the nasal mucosa by real-time PCR and western blotting. Viral modulation of the inhibitory molecules PD-L1 and PD-L2 in the nasal mucosa suppresses or terminates immune responses. PD-L1 exerts an inhibitory effect on type 1 immune responses, whereas PD-L2 inhibits type 2 responses and stimulates the type 1 response required for effective antiviral immunity $[11,24,25]$. In our study, HRV infection increased PD-L1 levels in the allergy group and decreased PD-L2 levels in both groups. This result implies that HRV infection could enhance the Th2 environment via the programmed cell death-ligand circuit and is related to the weakening of the antiviral immune response in AR patients.

To compare the susceptibility and reactivity to viral infection in the allergy and non-allergy groups, we investigated the expression of ICAM-1, the cell surface receptor for HRV. Some studies have reported that the expression of ICAM-1 was upregulated by HRV infection $[9,26]$.The allergic environment increases the expression of pattern-recognition receptor and ICAM-1
[26]. Our study did not reveal a difference in the ICAM-1 expression between the allergy and non-allergy groups (Fig. 2). ICAM-1 expression was elevated in CRS, and an allergy could enhance ICAM-1 expression, thereby inducing susceptibility to HRV infection [27]. Thus, in our study, pre-existing inflammation owing to CRS could augment the increased expression of ICAM1, implying that any further effect on ICAM-1 by HRV infection or allergic status would be minimal.

We identified the manner in which HRV infection affected the levels of IL-4, IL-5, IL-10, and IFN- $\gamma$ in the allergy and non-allergy groups. According to our results, HRV infection significantly increased the expression of IL-10 in the allergy group and that of IL-4, IL-5, and IFN- $\gamma$ in the non-allergy group. This does not mean that the levels of IL-4 and IL- 5 in the HRV-infected tissues from the non-allergy group were higher than those in the tissues from the allergy group. When the allergy and non-allergy groups were compared, the control tissue from the allergy group showed significantly higher mRNA levels of IL-4 and IL-5. Interestingly, the responses to HRV infection were different between the allergy and non-allergy groups. On the one hand, the IL-4/IFN- $\gamma$ ratio in the non-allergy group significantly increased after HRV infection; on the other hand, the ratio in the allergy group did not change significantly. Our data suggest that non-allergic subjects have an elevated risk of developing a Th2-biased environment after rhinovirus infection via the regulation of the Th2/Th1 balance.

When the nasal mucosa was infected with HRV, the expression of PD-L1 and IL-10 was elevated in the allergy group. Some studies have reported results similar to ours. Viral infection in the allergy group induced the expression of PD-L1, which is a selective co-stimulator of IL-10 secretion in both human and mouse T cells [12]. To determine the association between PD-L1 and IL-10, we conducted the Spearman correlation coefficient test in the allergy and non-allergy groups. A strong correlation was found between PD-L1 and IL-10 in the allergy group ( $r=$ $0.868, P<0.001)$; however, no correlation was evident in the non-allergy group ( $r=-0.018, P=0.973)$ (Fig. 4). Further studies are needed to elucidate the manner in which allergy factors work in the pathway underlying the PD-L1-mediated increases in IL-10. Our study suggests that HRV infection enhances the Th2 environment by modulating PD-L1 and PD-L2 expression levels in allergic mucosa and by increasing the IL-4/IFN- $\gamma$ ratio in non-allergic mucosa.

Our study presents data on the responses of patients with allergies involving chronic inflammation to HRV infection. To assess differences in the infection rate and immune responses based on allergic status only, HRV infection should be induced in normal controls and in patients with AR rather than in patients with CRS. Future studies should also include target groups that are homogeneous in terms of the CRS type to account for differences in immune mechanisms according to the type of CRS. In conclusion, this study shows that, regardless of the allergy condi- 
tion, the HRV infection rate was similar in the nasal mucosa of patients with CRS. In addition, HRV infection of the nasal mucosa from CRS patients appeared to enhance the Th2 environment by a different mechanism depending on allergic status.

\section{CONFLICT OF INTEREST}

No potential conflict of interest relevant to this article was reported.

\section{ACKNOWLEDGMENTS}

This research was supported by the Seoul National University Bundang Hospital research fund (grant no. 02-2012-038).

\section{ORCID}

Young-Kyung Ko

Yu-Lian Zhang

https://orcid.org/0000-0002-6139-4547

Jee Hye Wee

https://orcid.org/0000-0002-4079-8155

Doo Hee Han

https://orcid.org/0000-0002-6513-7011

https://orcid.org/0000-0003-3367-1495

Hyun-Jik Kim

https://orcid.org/0000-0001-8631-928X

Chae-Seo Rhee

\section{AUTHOR CONTRIBUTIONS}

Conceptualization: YKK, YLZ, JHW, CSR. Data curation: YKK, YLZ. Formal analysis: YKK, YLZ. Funding acquisition: JHW, CSR. Methodology: all authors. Project administration: YKK, DHH, HJK, CSR. Visualization: YKK. Writing-original draft: YKK. Writing-review \& editing: all authors.

\section{REFERENCES}

1. Jacobs SE, Lamson DM, St George K, Walsh TJ. Human rhinoviruses. Clin Microbiol Rev. 2013 Jan;26(1):135-62.

2. Pomeranz G, Pando R, Hindiyeh M, Sherbany H, MeningherT, Sharabi $\mathrm{S}$, et al. Rhinovirus infections in infants suggest that early detection can prevent unnecessary treatment. J Clin Virol. 2019 Jun;115: 11-7.

3. Drysdale SB, Mejias A, Ramilo O. Rhinovirus: not just the common cold. J Infect. 2017 Jun;74 Suppl 1:S41-6.

4. Proud D. Role of rhinovirus infections in asthma. Asian Pac J Allergy Immunol. 2011 Sep;29(3):201-8.

5. Juhn YJ. Risks for infection in patients with asthma (or other atopic conditions): is asthma more than a chronic airway disease? J Allergy Clin Immunol. 2014 Aug;134(2):247-57.

6. Singh AK, Stock P, Akbari O. Role of PD-L1 and PD-L2 in allergic diseases and asthma.Allergy. 2011 Feb;66(2):155-62.

7. Kortekaas Krohn I, Bobic S, Dooley J, Lan F, Zhang N, Bachert C, et

al. Programmed cell death-1 expression correlates with disease severity and IL-5 in chronic rhinosinusitis with nasal polyps. Allergy. 2017 Jun;72(6):985-93.

8. Deppong C, Juehne TI, Hurchla M, Friend LD, Shah DD, Rose CM, et al. Cutting edge: $\mathrm{B}$ and T lymphocyte attenuator and programmed death receptor- 1 inhibitory receptors are required for termination of acute allergic airway inflammation. J Immunol. 2006 Apr;176(7): 3909-13.

9. Zdrenghea MT, Johnston SL. Role of PD-L1/PD-1 in the immune response to respiratory viral infections. Microbes Infect. 2012 Jun; 14(6):495-9.

10. Akbari O, Stock P, Singh AK, Lombardi V, Lee WL, Freeman GJ, et al. PD-L1 and PD-L2 modulate airway inflammation and iNKT-celldependent airway hyperreactivity in opposing directions. Mucosal Immunol. 2010 Jan;3(1):81-91.

11. Ishiwata K, Watanabe N, Guo M, Tomihara K, Brumlik MJ, Yagita H, et al. Costimulator B7-DC attenuates strong Th2 responses induced by Nippostrongylus brasiliensis. J Immunol. 2010 Feb;184(4):208694.

12. Said EA, Dupuy FP,Trautmann L, Zhang Y, ShiY, El-Far M, et al. Programmed death-1-induced interleukin-10 production by monocytes impairs CD4+T cell activation during HIV infection. Nat Med. 2010 Apr;16(4):452-9.

13. Kosaka S, Tamauchi H,Terashima M, Maruyama H, Habu S, Kitasato H. IL-10 controls Th2-type cytokine production and eosinophil infiltration in a mouse model of allergic airway inflammation. Immunobiology. 2011 Jul;216(7):811-20.

14. Khan AR, Hams E, Floudas A, Sparwasser T, Weaver CT, Fallon PG. PD-L1hi B cells are critical regulators of humoral immunity. Nat Commun. 2015 Jan;6:5997.

15. Koya T, Matsuda H,Takeda K, Matsubara S, Miyahara N, Balhorn A, et al. IL-10-treated dendritic cells decrease airway hyperresponsiveness and airway inflammation in mice. J Allergy Clin Immunol. 2007 May;119(5):1241-50.

16. Wang JH, Kwon HJ, Chung YS, Lee BJ, Jang YJ. Infection rate and virus-induced cytokine secretion in experimental rhinovirus infection in mucosal organ culture: comparison between specimens from patients with chronic rhinosinusitis with nasal polyps and those from normal subjects. Arch Otolaryngol Head Neck Surg. 2008 Apr;134(4): 424-7.

17. Rhee CS, Min YG, Lee CH, Kwon TY, Lee CH, Yi WJ, et al. Ciliary beat frequency in cultured human nasal epithelial cells. Ann Otol Rhinol Laryngol. 2001 Nov;110(11):1011-6.

18. Kloepfer KM, Olenec JP, LeeWM, Liu G,Vrtis RF, Roberg KA, et al. Increased H1N1 infection rate in children with asthma. Am J Respir Crit Care Med. 2012 Jun;185(12):1275-9.

19. Santillan Salas CF, Mehra S, Pardo Crespo MR, Juhn YJ.Asthma and severity of 2009 novel H1N1 influenza: a population-based case-control study. J Asthma. 2013 Dec;50(10):1069-76.

20. Holt PG, Sly PD. Viral infections and atopy in asthma pathogenesis: new rationales for asthma prevention and treatment. Nat Med. 2012 May;18(5):726-35.

21. Caliskan M, Bochkov YA, Kreiner-Moller E, Bonnelykke K, Stein MM, $\mathrm{Du} \mathrm{G}$, et al. Rhinovirus wheezing illness and genetic risk of childhood-onset asthma. N Engl J Med. 2013 Apr;368(15):1398-407.

22. DeMore JP,Weisshaar EH,Vrtis RF, Swenson CA, Evans MD, Morin A, et al. Similar colds in subjects with allergic asthma and nonatopic subjects after inoculation with rhinovirus-16. J Allergy Clin Immunol. 2009 Aug;124(2):245-52.

23. Kim WK, Gern JE. Updates in the relationship between human rhinovirus and asthma. Allergy Asthma Immunol Res. 2012 May;4(3): 116-21.

24. Matsumoto K, Fukuyama S, Eguchi-Tsuda M, NakanoT, Matsumoto T, Matsumura M, et al. B7-DC induced by IL-13 works as a feedback 
regulator in the effector phase of allergic asthma. Biochem Biophys Res Commun. 2008 Jan;365(1):170-5.

25. Matsumoto K, Kan-O K, Eguchi-Tsuda M, Fukuyama S, AsaiY, Matsumoto T, et al. Essential role of B7-H1 in double-stranded RNA-induced augmentation of an asthma phenotype in mice. Am J Respir Cell Mol Biol. 2011 Jul;45(1):31-9.

26. Herbert C, Do K, ChiuV, Garthwaite L, Chen Y,Young PM, et al. Al- lergic environment enhances airway epithelial pro-inflammatory responses to rhinovirus infection. Clin Sci (Lond). 2017 Mar;131(6): 499-509.

27. Oyer SL, NagelW, Mulligan JK. Differential expression of adhesion molecules by sinonasal fibroblasts among control and chronic rhinosinusitis patients. Am J Rhinol Allergy. 2013 Sep-Oct;27(5):381-6. 